\title{
Parietal Lobe and Episodic Memory: Bilateral Damage Causes Impaired Free Recall of Autobiographical Memory
}

\author{
Marian E. Berryhill, ${ }^{1,2}$ Lisa Phuong, ${ }^{1}$ Lauren Picasso, ${ }^{1}$ Roberto Cabeza, ${ }^{3}$ and Ingrid R. Olson ${ }^{1,2}$ \\ ${ }^{1}$ Center for Cognitive Neuroscience, University of Pennsylvania, Philadelphia, Pennsylvania 19104, ${ }^{2}$ Department of Psychology, Temple University, \\ Philadelphia, Pennsylvania 19104, and ${ }^{3}$ Department of Psychology and Neuroscience, Duke University, Durham, North Carolina 27708
}

\begin{abstract}
Does the parietal lobe have a critical role in memory? The neuroimaging literature indicates that it has an important role, especially in episodic memory. However, the neuropsychological literature suggests that its role is more limited to attentional, spatial, or imagery aspects of memory. Here, we present data to adjudicate this disagreement. Two patients with bilateral parietal lobe damage received detailed assessments of their autobiographical memories. The results show that although both patients easily recalled various memories, their freely recalled memories were relatively impoverished, lacking in detail. This deficit was ubiquitous, and not limited to spatial or perceptual aspects of memory. The memory deficit disappeared when memory was specifically probed by asking pointed questions. Additional tests show that it is unlikely that their free recall deficit can be explained by general mental imagery problems. In sum, the parietal lobe appears to have a critical role in recollection aspects of episodic memory.
\end{abstract}

Key words: simultanagnosia; parietal; autobiographical memory; imagery; memory; neuropsychology

\section{Introduction}

The human parietal lobe has historically been linked to linguistic, visual, and visual-motor functions. Classic texts describing parietal function make no reference to mnemonic functions and indeed, it would surprise many neurologists to hear that the parietal lobe had any role in memory (Critchley, 1953; Luria, 1966).

Although memory deficits are not traditionally associated with parietal damage, there are two notable (and related) exceptions. First, reports link parietal lobe damage, usually in the context of aphasia, to verbal short-term memory (STM) deficits (Ghent et al., 1962; Warrington et al., 1971; De Renzi and Nichelli, 1975; Risse et al., 1984) (for review, see D'Esposito and Postle, 1999). Second, right parietal lobe damage, in the context of hemispatial neglect, leads to spatial STM impairments (Husain et al., 2001; Wojciulik et al., 2001; Pisella et al., 2004; Malhotra et al., 2005). One might argue that observed STM deficits are not truly mnemonic, but rather, arise from the reliance of spatial STM on spatial attention (Baddeley et al., 1984; Craik et al., 1996), which is damaged. However, this argument is weakened by new evidence showing that right parietal damage, in the absence of hemispatial neglect, causes STM deficits for visual features such as color and shape, as well as for location (Berryhill and Olson, 2007).

These findings demonstrate parietal involvement in short-

Received July 11, 2007; revised Nov. 19, 2007; accepted Nov. 20, 2007.

This work was supported by National Institutes of Health Grant R01 MH071615-01 (I.R.0.). We thank Brian Levine for the use of his training and scoring materials, Morris Moscovitch for helpful advice, Branch Coslett for theoretical discussions, Junhyong Kim for providing statistical assistance, and patients EE555 and TQ591 for their time.

Correspondence should be addressed to Marian E. Berryhill, Center for Cognitive Neuroscience, University of Pennsylvania, 3810 Walnut Street, Philadelphia, PA 19104-6196. E-mail: berryhi@@psych.upenn.edu.

DOI:10.1523/JNEUROSCI.4163-07.2007

Copyright $\odot 2007$ Society for Neuroscience $\quad$ 0270-6474/07/2714415-09\$15.00/0 term forms of memory, but what about longer-term forms of memory? Here, neuropsychology is silent, as little is reported, whereas neuroimaging is vocal. Parietal cortices are frequently activated in neuroimaging studies of episodic memory retrieval (Cabeza and Nyberg, 2000; Wagner et al., 2005). Posterior parietal cortex (PPC) regions show greater activity during correct recognition of old items than during correct recognition of new items (Henson et al., 1999; Eldridge et al., 2000; Konishi et al., 2000; Cabeza et al., 2001). Moreover, neuroimaging evidence suggests that different PPC subregions contribute to different memory processes. In particular, inferior, also referred to as ventral PPC (angular gyrus and temporoparietal junction) shows greater activity for retrieval accompanied by vivid episodic details (recollection) than for the feeling that an item occurred in the past in the absence of specific details (familiarity) (Henson et al., 1999; Eldridge et al., 2000; Wheeler and Buckner, 2004; Yonelinas et al., 2005; Daselaar et al., 2006). In contrast, familiarity has been associated with more dorsal PPC (Wheeler and Buckner, 2004; Yonelinas et al., 2005) and occipitoparietal activations (Daselaar et al., 2006).

The link between ventral PPC and recollection fits well with the results of functional neuroimaging studies of autobiographical memory, in which participants retrieve memories from their own life (for review, see Svoboda et al., 2006; Cabeza and St Jacques, 2007). The parietal regions most frequently activated in these studies are located in the vicinity of Brodmann's Area 39 (Conway et al., 1999; Maguire and Mummery, 1999; Nyberg et al., 2002; Addis et al., 2004; Gilboa et al., 2004; Levine et al., 2004), including the angular gyrus and temporoparietal junction.

These functional neuroimaging findings strongly linking PPC to memory raise an interesting question: why are reports of episodic memory deficits in patients with parietal lobe lesions difficult to find? One plausible answer is that parietal lesions do not 
Table 1. Neuropsychological evaluation of patients EE555 and TQ591

\begin{tabular}{llcc}
\hline Test & Subtest & EE555 & TQ591 \\
\hline AMI & Personal semantic & 49.5 & 60 \\
\multirow{3}{*}{ WMS } & Autobiographical incidents & 21 & 17 \\
& Immediate & 86 & 80 \\
& Delayed & 77 & 97 \\
& WM & 83 & 79 \\
\multirow{2}{*}{ WAB } & Recognition delayed & 55 & 110 \\
& Auditory verbal comprehension & 60 & 60 \\
& Sequential Commands & 80 & 76 \\
& Repetition & 100 & 98 \\
\hline
\end{tabular}

The AMI personal semantic score is out of a total of 63 (normal, 54-63); the autobiographical semantic measure is out of a total of 27 (normal, 19-27). WMS scores are index scores from auditory subtests in which the population mean is 100 and the SD is 15 . We consider performance on the WMS and AMI abnormal if it deviates from the population mean by 1 SD. The WAB measures were scored out of 60,80 , and 100 possible points. WM, Working memory. Abnormal scores are italicized.

impair episodic memory. This possibility is not inconsistent with functional neuroimaging evidence, because neuroimaging evidence only proves that parietal regions are involved in episodic, not that they are necessary for this function. A second answer is that parietal lesions do impair episodic memory but these deficits are subtle. Episodic memory deficits might be concealed by perceptual and language deficits associated with parietal lesions, and/or because they are specific to certain forms of episodic memory and noticed only after thorough evaluation. Thorough memory testing is seldom performed in parietal patients because it is assumed these patients do not have memory deficits. The goal of the present study was to examine whether the second answer is true.

Autobiographical memory was tested because it minimizes encoding differences between patients and controls because most of the recounted memories preceded neurological insult. As such, the present findings do not address parietal lobe involvement in memory encoding. Our initial neuropsychological evaluation of memory (see Materials and Methods) (see Table 1) showed that both patients performed poorly on several tests of memory, including one test of autobiographical memory. Thus, we performed a second, more detailed, examination of autobiographical memory using Levine's Autobiographical Memory Interview (Levine, 2004) in which autobiographical memories are freely recalled and subjected to detailed textual analysis. Last, we assessed the relationship between autobiographical memory and visual imagery.

\section{Materials and Methods Participants}

Lesion patients

Two patients with bilateral parietal lobe damage were tested in this study. Table 1 summarizes their performance on various perception and neuropsychological tests.

\section{Patient EE555}

EE555 is a 39-year-old former teacher with 16 years of education. Between April and June, 2004, she suffered three infarcts in the watershed between the posterior and middle cerebral arteries. Acute symptoms included intense headaches and complete blindness, which was temporary. After her third stroke, she was admitted to the Hospital of the University of Pennsylvania where she was treated for hypertension, and anatomical magnetic resonance imaging (MRI) scans were performed. The initial neurological evaluation revealed alexia and canonical symptoms of simultanagnosia, symptoms of which are detailed in Table 1.

EE555's physical and perceptual symptoms are currently stable. Her MRI revealed symmetrical lesions in lateral aspects of the inferior parietal lobe, extending from superior aspects of the occipital lobe through the angular gyrus [Brodmann's area (BA) 39] in and around inferior and middle portions of the intraparietal sulcus (IPS). Damage did not encroach into the midline (e.g., precuneus). EE555's lesions are depicted in Figure 1.

Patient EE555's primary deficit is simultanagnosia. When shown a line drawing of a visual scene, she describes parts of the picture, "there is a woman," and "I see water," without attaining a global understanding of the scene. In line cancellation tasks, she crosses off items only at the center, ignoring peripheral items. She only reports the local elements when shown Navon letters. She also suffers from optic ataxia, misreaching and mispointing to foveated and peripheral objects. She does not have optic apraxia (inability to change location of fixation), nor does she suffer from left-right confusion. Language comprehension and speech fluency were unimpaired as assessed by her conversational skills, and by ceiling performance on the auditory tests of the Western Aphasia Battery. Reading and writing are impaired because of her simultanagnosia and spatial disorientation (she loses her place on a page and commits mirror reversal errors in writing). Her eyesight is normal. Object and color perception are normal as measured by a matching task ( score $=100 \%)$.

\section{Patient TQ591}

TQ591 is a 49-year-old former preschool assistant teacher with 15 years of education. She suffered bilateral parieto-occipital damage caused by CNS cerebral vasculitis in March 2006 and was treated at the Hospital of the University of Pennsylvania.

TQ591's MRI revealed signs of previous subacute posterior cerebral artery infarctions. The primary lesions are in bilateral parietal regions (Fig. 1). The left parietal lesion extends into IPS (BA 39) and precuneus (BA 7). There are two right lesion sites: the inferior lesion is in superior aspects of the occipital lobe (BA 18 and 19), and the superior lesion is in the superior parietal lobe (BA 7). In both hemispheres, the lesions extend slightly into temporo-occipital (BA 19) regions and parietal white matter.

TQ591's primary deficit is simultanagnosia. When shown pictures of scenes, TQ591 is slow to describe them and complains that parts of scenes 'disappear' when she looks away or blinks and she cannot relocate them. In line cancellation tasks, she only identifies a few lines within a narrow visual field. When asked to describe a computer keyboard, TQ591 reported seeing the " $\mathrm{H}$ " key and nothing else, after a deliberate blink, she reported seeing only the "G" key and nothing else. She has a local bias with Navon letters. She suffers from mild optic ataxia, misreaching to objects in the periphery but not in the fovea. She also suffers from optic apraxia, making it effortful for her to move her eyes from where they are looking. One strategy she employs is to blink to release her attention. She also has mild left-right confusion.

Language comprehension and speech fluency were unimpaired as assessed by her conversational skills, and ceiling performance on the auditory tests of the Western Aphasia Battery. Reading and writing were somewhat impaired because of her simultanagnosia and spatial disorientation (she loses her place on a page). Her vision is corrected-to-normal. Object and color perception are normal as measured by a matching task $($ score $=100 \%)$.

In summary, the pattern of deficits and preserved abilities found in these patients are in line with Balint's classic report of bilateral parietal lobe damage. Both patients are highly personable, alert and attentive participants.

\section{Neuropsychological evaluation of memory}

To attain a general overview of the patient's memory abilities, several neuropsychological tests were administered. First, to establish that patients are able to follow verbal instructions we conducted auditory subtests of the Western Aphasia Battery (WAB-R; Harcourt Assessment, San Antonio, TX). Auditory subtests of the Wechsler Memory Scale (WMS-III; The Psychological Corporation, San Antonio, TX) were administered. EE555 performed at least 1 SD below the mean on all tests, whereas TQ591 was impaired on the immediate memory and working memory components (see Table 1). It should be noted that medial temporal lobe amnesics generally score at least two SD below the mean. EE555's "recognition delayed" score fell into this range.

As an initial assessment of parietal involvement in autobiographical 


\section{EE555}
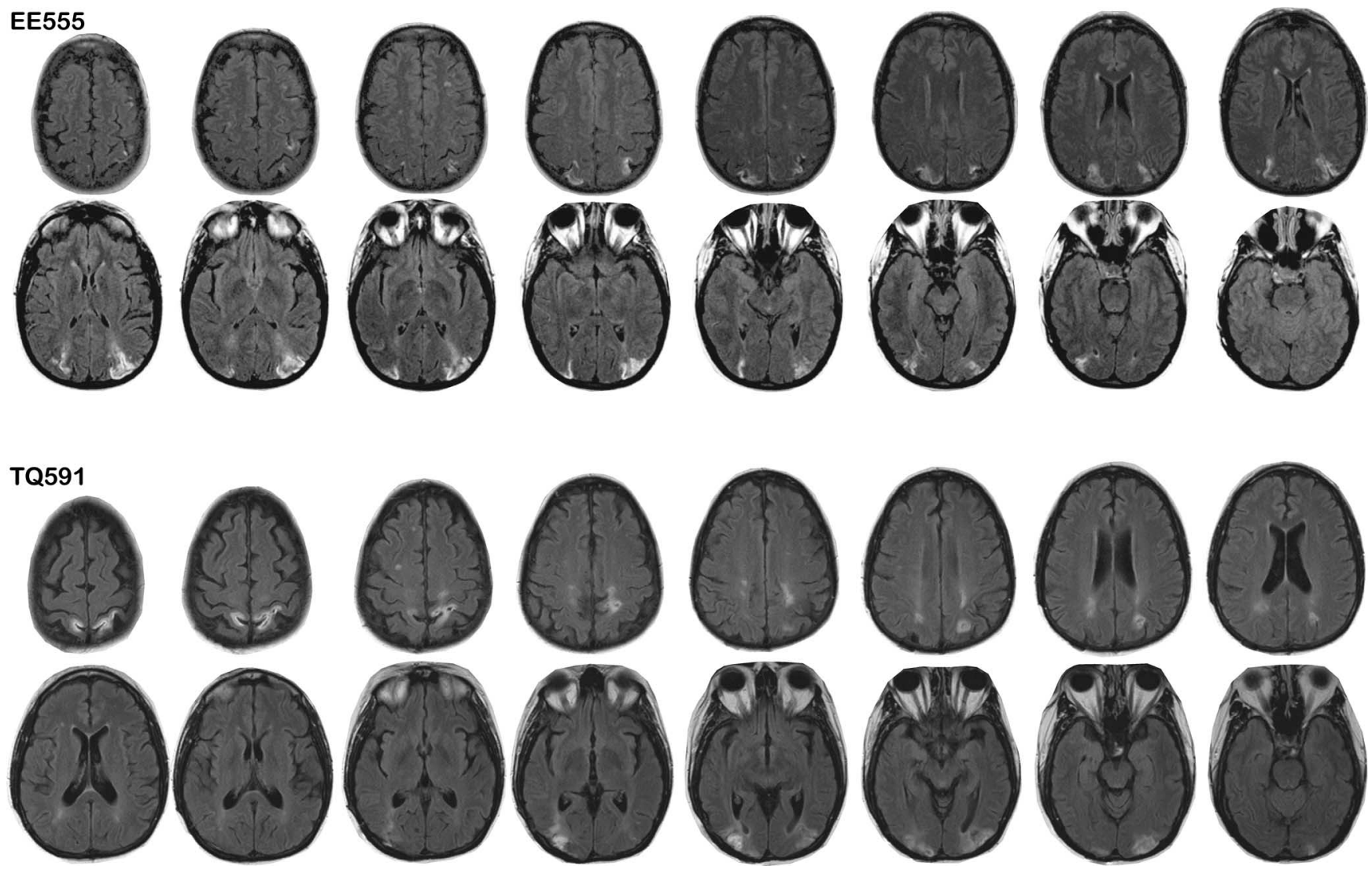

Figure 1. Patient lesion traces. Lesions are shown on $\mathrm{T} 2$ fluid-attenuated inversion recovery images in which the lesions appear as white higher intensity patches in parietal regions. The right is on the left.

memory, a standard autobiographical memory test, the autobiographical memory inventory (AMI) (Kopelman et al., 1989), was administered. This test measures memory for personal semantic and autobiographical incidents in a short-answer format. The results showed that both patients were subtly abnormal on this test. EE555's semantic recollections were scored as "probably abnormal" (see Table 1), but her description of autobiographical events was in the "acceptable" range. TQ591 showed the reverse pattern. In summary, these findings provide one hint that the parietal lobe is involved in autobiographical memory.

\section{Control participants}

Ten age- (mean, 46.7; range, 37-57, 6 males) and education- (mean, 15.0; range, 10-19) matched normal controls participated in experiment 1 . Ten age- (mean, 48.8; range, 35-62, 5 males) and education- (mean, 14.2 ; range, 12-18) matched controls participated in experiment 2 . There were no differences between patients and controls in terms of age or education in either experiment (all $p$ values $>0.20$ ).

\section{Experiment 1: autobiographical memory retrieval}

Levine et al. (2004) previously published an elaborate memory test designed to assess autobiographical memory in fine detail by capturing the richness of self-related memory. This test employs text analysis of subjects' freely reported memories to examine the level of detail in descriptions of autobiographical events. Text analysis is also used to examine the semantic manner in which subjects describe their memories. After the free report portion of the test, specific probe questions are asked to guide the subjects' responses. We predicted that the bilateral patients would describe their autobiographical memories with less vividness and fewer perceptual and spatial details, given their impoverished perception of space.

\section{Procedure}

The procedure strictly follows the methods provided by Rosenbaum et al. (2004) and detailed by Levine et al. (2002), which are summarized here.
Participants were asked to recall significant events from five life periods: early childhood (up until age 10), adolescent years (ages 11-17), early adulthood (18-34), middle age (35-55), and the previous year. Age ranges for the early adulthood and middle age were shortened for one younger control participant $(18-29,30-35$, respectively) to define five time periods. To assist with memory retrieval, we provided auditory cues of typical life events (see supplemental Materials and Methods, available at www.jneurosci.org as supplemental material) if the participant did not immediately begin recounting autobiographical events. For each time period, participants were queried under three conditions: free recall, general probe, and specific probe.

During free recall, participants verbally described an event extemporaneously without interruption, until they reached a natural ending point. After freely recalling a memory, general probes were used to clarify instructions and to encourage greater recall of details if they did not recall a specific event or if recollection was too brief (i.e., "Can you tell me a more specific instance?" or "Is that everything you can say about it?"). These two steps were repeated for a single event from each of five life periods. Finally, the experimenter followed-up each event with a series of specific probe questions, following the methods of Levine et al. (2004), which consists of a structured interview adapted from the Memory Characteristics Questionnaire (Johnson et al., 1988). This list of 17 probe questions is designed to evoke overlooked details and focus attention on the details related to the precise time of an event, location, perceptual details, and emotional reactions (see supplemental Materials and Methods, available at www.jneurosci.org as supplemental material).

The entire interview was audio recorded with GarageBand software (Apple Computer, Cupertino, CA) and then transcribed. After transcription, two trained, blind scorers with high inter-rater reliability, as confirmed by correlation analysis $(r=0.93)$, rated each memory based on instructions and a manual provided by Levine et al. (2002). The chief dependent measure was the tallied number of details. Recollected details pertaining to the probed event are considered internal details, and were 
categorized as relating to: event, place, time, perceptual, and emotion/ thought. Recollected details that were irrelevant to the probed event (e.g., asides, wanderings, musings, etc.), are considered external details, and were placed in one of eight categories (event, place, time, perceptual, thought, semantic facts, repetitions, or other metacognitive statements). There were few references to place, time, or other metacognitive statements, leading us to collapse across these categories and refer to them as "other."

A second dependent measure of memory rating was subjectively determined following published guidelines (Levine et al., 2002) to provide a gestalt sense of memory quality. The two raters scored each recollected memory on a scale of $0-3$ ( 3 being the highest score) based on the vividness of each internal category. A score of 3 was given if the memory description provided a sense of re-experiencing the event in a particular place and context. A score of 2 was given if there was a lesser degree of detail. A score of 1 was given if general, less-specific information was supplied and a score of 0 was given if no specific information or only semantic knowledge was expressed. Following the method of (Levine et al., 2002), the episodic richness scale was evaluated using identical criteria, except the scale was expanded to a six-point scale to provide greater specificity in measurement.

\section{Statistical analysis}

The dependent measures in experiment 1 were the number and type of details retrieved from memory. The task requires a mixed two-factor design for which a standard nonparametric test is unavailable. For these data, a permutation test was used in which we first computed the $F$ statistic under the standard mixed two-factor ANOVA model. Then the observed values were randomly permuted across the patient and control subjects. The $F$ statistics were recomputed for the permuted data set and a one-tailed count over 1000 replicates was used to compute the significance values (Legendre et al., 1990; Manly, 1997). To assess which level of a factor was driving the main effect, pairwise comparisons using the Mann-Whitney $U$ test were conducted and the z-scores are reported because they are more familiar than the $U$ values. The significance values reported are the two-tailed values provided using SPSS software (SPSS, Chicago, IL).

In experiment 2, the dependent measure was response accuracy. Man$\mathrm{n}$-Whitney tests were conducted as the nonparametric alternative to independent samples $t$ tests.

\section{Experiment 2: spatial and nonspatial imagery}

To assess whether our patients had general or spatial-specific mental imagery deficits, spatial and nonspatial mental imagery of all subjects was assessed, using various tests that have been successfully administered to neuropsychological patients in the past (Farah et al., 1985, 1988; Kosslyn et al., 1985; Levine et al., 1985; Rosenbaum et al., 2004). In particular, we closely followed an approach used to test a well known amnesic, patient K.C. (Rosenbaum et al., 2004). If the bilateral parietal patients have a general mental imagery deficit, much like the general free recall problem observed on the memory test, they should be impaired on all of the tasks, regardless of whether there is spatial component.

\section{Tests of nonspatial visual imagery}

Letter shape test. Based on the imagery test created by Farah et al. (1985), letters were spoken one at a time. Participants imagined the uppercase version of each letter and determined whether the letter was formed with purely straight lines or whether there were also curved lines (e.g., A vs B).

Tail judgment test. A list of 20 animals were read aloud one at a time. Participants determined whether the animal has a long or short tail in relation to its body (Farah et al., 1988). As a control task the subjects also reported the animal's typical color.

Object pairs test. Sixteen pairs of objects of similar size were read aloud, one object pair at a time (Farah et al., 1988). Participants determined which of the two objects was larger (e.g., couch and hammock).

\section{Tests of spatial imagery}

Mental clock test. A list of 24 times were read aloud one at a time. Participants determined whether the angle of the hour and minute hands was
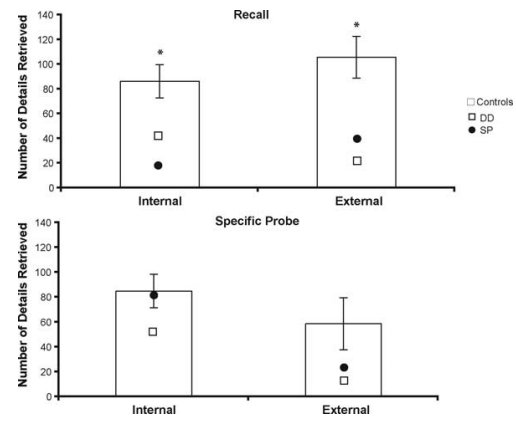

Figure 2. Internal and external details retrieved during free recall of memories (top) and when specifically probed (bottom). Internal details refer to details pertaining to the probed event. External details refer to details that were irrelevant to the probed event. Error bars represent SEM. Asterisks denote significant differences between the control and patient groups.

smaller than $90^{\circ}$ (Paivio, 1978; Craik and Dirkx, 1992; Rosenbaum et al., 2004).

Brooks letter test. The participant was asked to imagine walking a path around a large capital letter E and F (Brooks, 1968; Rosenbaum et al., 2004). The participant was asked to report each turn starting from the lower left corner of the letter and making a full circuit. There were 20 turns in total.

Verification of high-and low-imagery sentences. The participant heard high (16) and low (16) imagery sentences and made true-false judgments as to their veracity. This task is designed to tax both object and spatial visual imagery and compare performance against similar sentences for which no imagery is required. Equal numbers of true and false statements were read (Eddy and Glass, 1981).

\section{Results}

\section{Experiment 1}

\section{Performance by time period}

To evaluate performance as a function of time period, we collapsed across type of detail and performed permutation analyses. There was no significant interaction of group membership and time period for any measure (all $p$ values $>0.37$ ). In the following analyses, we collapsed across time period to improve statistical power.

\section{Free recall and specifically probed recall}

This analysis determined whether any differences between patients and controls could be attributed to shorter verbal recollections by the patients. The word counts for the patients (TQ591, 129.6; EE555, 200.2) and controls (mean, 324.2; SD, 210.0; range, 92.0-789.4) during the free recall portion were compared and no significant differences were found (Mann-Whitney test, $\mathrm{z}=$ $-1.50, p=0.13$ ). This suggests that differences between the two groups were not due to the patients simply uttering fewer words.

To see whether the parietal lobe is particularly important for retrieving details associated with the memory of interest (internal) as well as unrelated but recounted details (external) of autobiographical memories, the total number of internal and external details related during the free recall period were subjected to permutation analysis. The factors were group (patient, control) and type of detail (internal, external) (Fig. 2, top). The results show that bilateral parietal patients recounted relatively fewer details $\left(F_{(1,10)}=364.97, p=0.01\right.$; mean control, 96.08; mean patient, 31.38). There was no main effect of type of detail $\left(F_{(1,10)}\right.$ $=2.95, p=0.13$ ) and no interaction of group and type of detail $\left(F_{(1,10)}<1\right.$, not significant). In sum, the patients exhibited a global deficit in freely recalling internal and external details.

In contrast, when given specific probes to focus recollection (Fig. 2, bottom), the performance of the patients and controls did 
not differ $\left(F_{(1,10)}=42.75, p=0.39\right)$. As in the previous analysis, there was no effect of the type of detail $\left(F_{(1,10)}=3.49, p=0.10\right)$ and the interaction of group and type of detail was not significant $\left(F_{(1,10)}=1.07, p=0.40\right)$.

Free recall: analysis of performance in subcategories

It is possible that the poor performance of the bilateral parietal patients was the result of under-reporting of perceptual details, mirroring their visual perception deficits. If true, then recall deficits should be specific to recollection of perceptual information. To assess this, the free recall data for the internal detail category were categorized into five types of recollections (event related, time, place, perceptual and thought/emotion) following the approach of Levine et al. (2002). As can be seen in Figure $3 a$, the patients reported numerically fewer details in each category. However, certain categories were pathologically under-reported: Mann-Whitney tests showed that patients recalled significantly fewer time $(z=-1.96, p=0.05)$, perceptual $(z=-2.15, p=$ $0.03)$, and thought $(z=-2.05, p=0.04)$ internal details. The number of place related details approached significance $(z=$ $-1.83, p=0.07)$. The fifth category, event related details, was not significant $(z=-1.29, p=0.20)$.

A similar analysis was conducted on data from the external detail category although in this case the categories were: external event, semantic, repetition and other. As can be seen in Figure $3 b$, the patients reported numerically fewer details in each category. However, certain categories were pathologically underreported: Mann-Whitney tests showed that patients recalled significantly fewer details related to external events $(z=-1.93, p=0.05)$, external semantic details $(z=-2.15, p=0.03)$ and other (e.g., place, time, or other metacognitive statements) external details $(z=-2.16, p=0.03)$. The number of repetitions of external details failed to reach significance $(z=-1.3, p=0.20)$.

In a secondary analysis, a different dependent measure, the memory ratings subjectively given by the blind raters, were used to assess group differences over five detail categories (event related, time, place, perceptual and thought) (Fig 3c, top). Bilateral parietal patients were relatively impaired in four of the five categories (all $z$ values $>-1.94$, all $p$ values $<0.05$ ). The one comparison that was borderline significant was in the ratings of freely recalled perceptual details $(z=-1.83, p=0.07)$. In sum, these findings show that poor free recall of autobiographical memories is not limited to perceptual aspects of memories but rather, affects most aspects of autobiographical memory.

Specific probes: analysis of performance in subcategories

In Figure 2 it can be seen that bilateral parietal damage causes impaired autobiographical memory under free recall conditions but not under specific probe conditions. In post hoc analyses, we assessed whether there was any evidence of impaired memory in the specific probe data, for particular types of internal details, by breaking down the data into the five aforementioned categories. Mann-Whitney tests found that patients and controls performed similarly across all categories (all $p$ values $>0.10$ ) (Fig. $4 a$ ).

For external details, there was a numerical trend toward lower reporting of details across categories, although Mann-Whitney values failed to reach significance (all $p$ values $>0.10$ ) (Fig. $4 b$ ). In sum, however, there was no evidence of memory impairments in bilateral parietal patients when autobiographical memory is specifically probed.

A secondary analysis using the memory ratings given by the blind raters, which collapses across the internal and external categories, found that the patient's reported memories lacked epi-
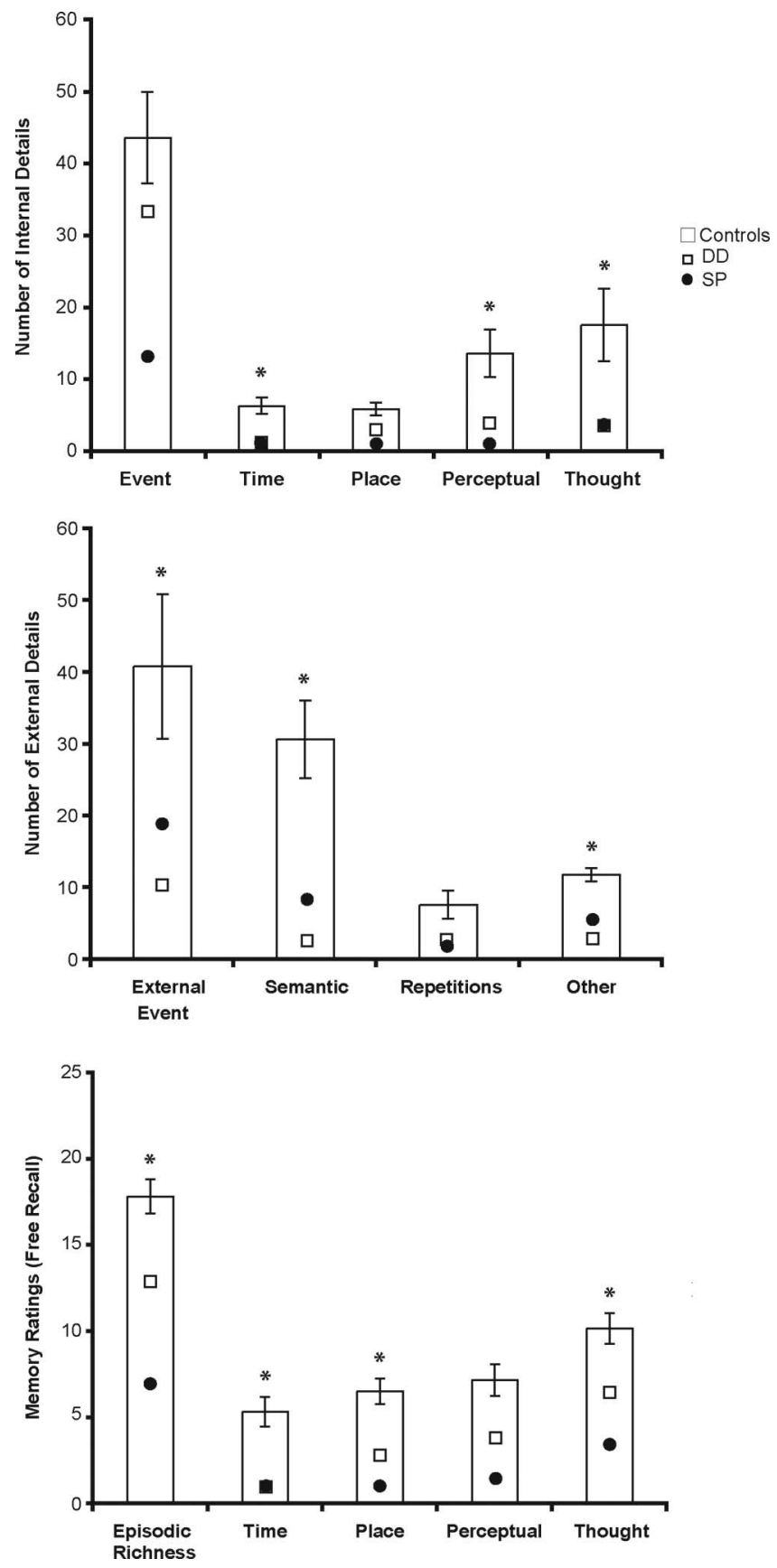

Figure 3. Free recall performance. Top, Number of internal details. Middle, External details. Bottom, Memory ratings for different categories retrieved during the free recall condition. The axes for the top and middle graphs reflect the total number of details tallied for each memory. The axes for the bottom graph reflect the scale of $0-6$ for episodic richness and $0-3$ for the remaining categories. Error bars represent SEM. Asterisks denote significant differences between the control and patient groups.

sodic richness $(z=-2.16, p=0.03)$ and thought/emotional content $(z=-2.16, p=0.03)$. The remaining three categories showed no trend toward significance (all $p$ values $>0.13$ ) (Fig. 4c).

These results show that bilateral parietal damage leads to impaired autobiographical memory in one recall condition, but not another. The patients freely recalled fewer details of episodic memories. Their deficit was not due to a statistically significant difference in the number of words uttered. However, this null finding may be partially due to lack of power because we assessed 

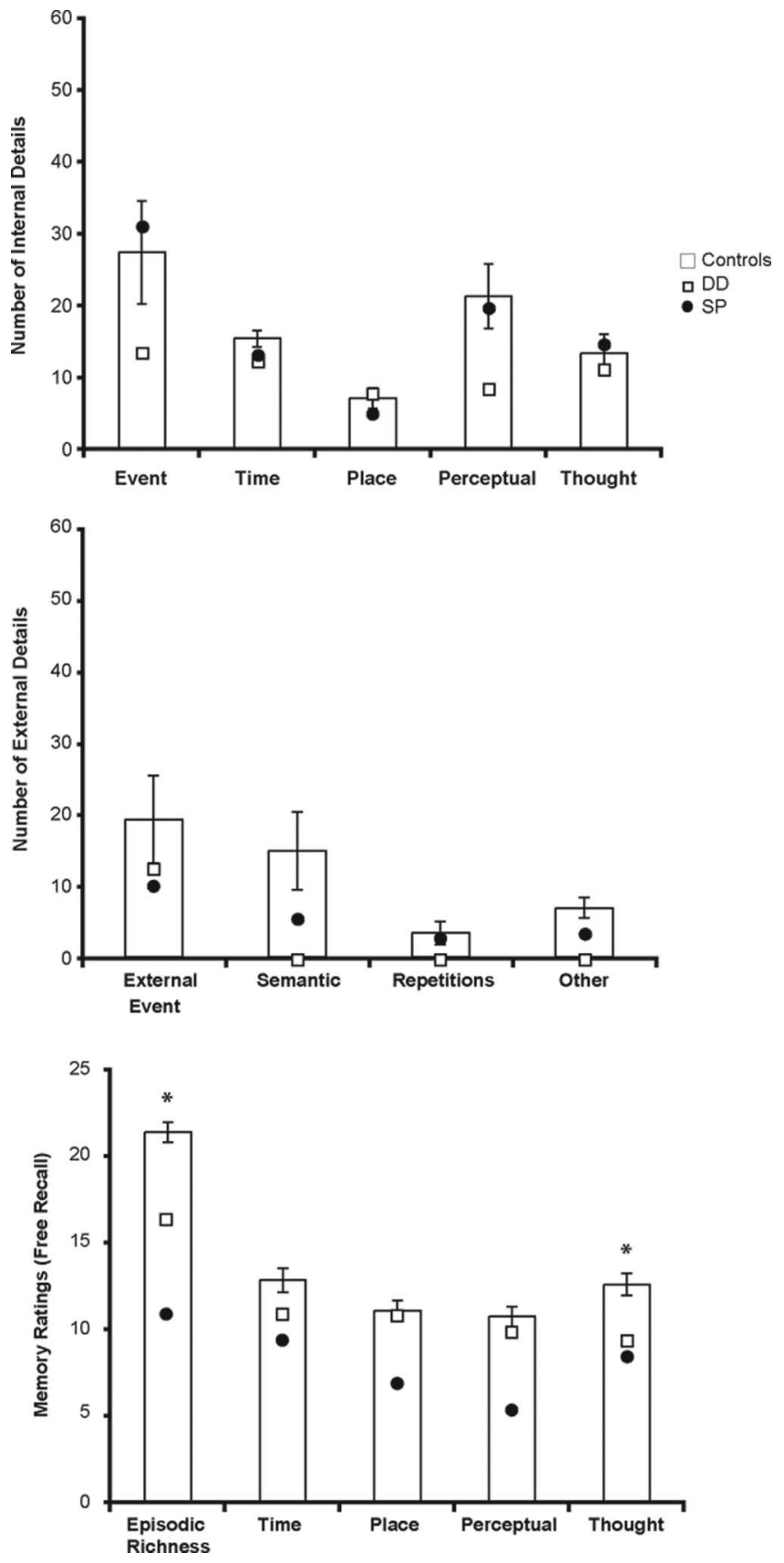

Figure 4. Specific probe performance. Top, Number of internal details. Middle, External details. Bottom, Memory ratings for each category in retrieved during the specific probe condition. The axes for the top and middle graphs reflect the total number of details tallied for each memory. The axes for the bottom graph reflect the scale of $0-6$ for episodic richness and $0-3$ for the remaining categories. Error bars represent SEM. Asterisks denote significant differences between the control and patient groups.

performance in two patients and patient TQ591 spoke fewer words per memory than the control subjects. Importantly, their deficit was not restricted to spatial or perceptual details, as one might expect with parietal lobe damage, but rather generalized over a variety of memory categories. This pattern of results was consistent across text analyses tallying the number of internal and external details. It was also noticeable in the more subjective memory rating analysis in which the experimenters used a Likert scale to rate each memory. However, when the patients are specifically probed for details relating to each memory they were able to report considerably more information than during the free report test. These findings suggest that the memories remain relatively intact and that their memory deficit is restricted particular memory retrieval problems.

One explanation for these findings is that the difficulty with free recall of life events falls under a more general impairment of mental imagery. Intact mental imagery may be a prerequisite for recalling vivid autobiographical memories. Case reports dating back to the $1940 \mathrm{~s}$ report that parietal lobe damage can at times impair spatial mental imagery but generally leaves object mental imagery intact (for review, see Tippett, 1992). However, it is possible that our patients have a more global mental imagery deficit which if found, would partially explain their impoverished free recall of self-related memories. To test this, we conducted experiment 2.

\section{Experiment 2}

Nonspatial visual imagery tasks

Letter shape task. The controls and patient TQ591 scored 20/20 on this test, whereas patient EE555 scored 18/20 (controls: mean, 100.00, SD, 0; patients: mean, 95.0\%; SD, 10.0; $z=-2.24, p=$ $0.03)$.

Tail task. TQ591 (16 of 20) and EE555 (18 of 20) performed no differently from controls when deciding whether the animal has a long tail relative to its body (controls: mean, 74.55\%; SD, 12.35; patients: mean, 89.44\%; SD, 0.79; $z=-1.09, p=0.28)$.

Animal color task. TQ591 (17 of 20) and EE555 (20 of 20) performed no differently from controls when making judgments about the color of the animal (controls: mean, 90.00\%; SD, 9.13; patients: mean, 97.22\%; SD, 3.93; $z=-0.45, p=0.13)$.

Mental size comparisons. TQ591 (13 of 20) and EE555 (12 of 20) performed no differently from controls when making size comparisons of similar objects (controls: mean, $78.13 \%$; $\mathrm{SD}=$ 7.37; patients: mean, 78.13\%, SD, $4.42 ; z=-0.11, p=0.91$ ).

\section{Spatial imagery tasks}

Clock face. TQ591 (13 of 20) and EE555 (14 of 20) were impaired at measuring the angle of clock hands using spatial imagery, but the controls were highly variable so that the difference between groups failed to reach statistical significance (controls: mean, 78.33\%; SD, 15.06; patients: mean, $56.25 \%$; SD, $2.95 ; z=-1.53$, $p=0.13)$.

E/F mental letter tracing task. TQ591 (5 of 20) and EE555 (0 of $20)$ were very impaired on this task (controls: mean, 85.00; SD, 19.27; patients: mean, 12.5 ; SD, 17.68; $z=-2.12, p=0.03$ ).

\section{Verification of high-and low-imagery sentences}

TQ591 (14 of 16) and EE555 (11 of 16) performed no differently from controls on the high imagery sentences (controls: mean, 88.28; SD, 13.54; patients: mean, 78.13; SD, 13.26; $z=-0.77, p=$ 0.44 ) or on the low imagery sentences (TQ591, 13 of 16; EE555, 13 of 16), although there was a trend toward lower performance by the patients (controls: mean, 92.97; SD, 7.04; patients: mean, 81.25; SD, $0 ; z=-1.78, p=0.08$ ).

In line with previous findings (for review, see Tippett, 1992), the results of Experiment 2 suggest that bilateral parietal lobe damage impairs spatial mental imagery, but not nonspatial mental imagery. These findings fail to support the hypothesis that the observed autobiographical memory deficits can be explicated by global mental imagery problems.

\section{Discussion}

The experiments reported here were conducted to clarify the role of the parietal lobe in episodic memory, specifically autobio- 
graphical memory. We chose to study autobiographical memory because it allowed us to reduce demands on sensory encoding processes because autobiographical memory encoding mostly occurred before neurological insult.

The results clearly indicate that the parietal lobe has a functional role in episodic, and more particularly, autobiographical memory. Experiment 1 evaluated autobiographical memory in detail and showed that the patients' recollections lacked richness and specificity when they were asked to freely recall memories from different points in time. Moreover, this deficit was not restricted to spatial or perceptual details of memories, but rather was found across all categories (Fig. 2). In contrast, the patients were not differentially impaired in their ability to answer probe questions relating to a particular memory as by the "specific probe" condition. There was a trend in the patient data to report fewer external details that were not central to the particular autobiographic memory.

What is the specific role of parietal cortex in memory? We approach this question by first ruling out some commonplace explanations. First, it is unlikely that the observed deficits had anything to do with memory encoding, because autobiographical encoding occurred almost entirely before neurological insult. Only autobiographical memories from the past year were encoded afterward. Second, the observed deficit is not specifically linked to recalling aspects of memories that might plausibly be linked to the patients' perceptual deficits (extraordinary problems with perceiving space and location) because the observed deficits were not specific to spatial or perceptual aspects of memories (Fig. 4). Last, the observed memory deficits are unlikely caused by deficient mental imagery because the patients did not demonstrate global mental imagery impairments. It has been reported that hippocampal damage impairs the ability to imagine future scenarios (Hassabis et al., 2007); this type of mental imagery was not assessed in the bilateral parietal patients. However, even if such deficits did exist, there is no logical reason why deficient constructive memory should account for autobiographical memory deficits. In sum, the pattern of results reported in Experiment 2 is consistent with past findings associating impaired spatial imagery, but intact nonspatial imagery, with dorsal stream damage (Bisiach and Luzzatti, 1978) (for review, see Farah, 1989; Tippett, 1992; Cavanna and Trimble, 2006; Byrne et al., 2007).

We also note that the patient's deficits were not caused by a language production deficit. We raise this issue because on average, the patients uttered numerically fewer words than controls, although statistically there was no significant difference. One explanation is for this is that the patients have a subtle language production deficit that would be more apparent with greater statistical power. However, the patients do not have language comprehension or production deficits as assessed by self report, neurological examination, or neuropsychological assessment. We think a simpler explanation is that when memories are sparse you simply have less to say.

The autobiographical memory findings reported here clearly indicate that the parietal lobe has an essential role in one particular component of memory: memory retrieval. Patients had impaired recall of internal details under free recall conditions but intact recall of internal details when specifically probed. It is for this reason that patients with parietal lobe damage do not appear to be amnesic: with the right set of cues, they can readily recollect past experiences. Interestingly, there was some evidence that their recall of external, or unrelated, details was impoverished under both free recall and probed recall conditions, although this failed to reach statistical significance.
Functional MRI studies of autobiographical retrieval have reported activations throughout the parietal cortex, including the angular gyrus, supramarginal gyrus, intraparietal sulcus and precuneus (Conway et al., 1999; Maguire and Mummery, 1999; Nyberg et al., 2002; Addis et al., 2004; Gilboa et al., 2004; Levine et al., 2004). Patient EE555's lesions include damage to the angular gyrus but do not extend into supramarginal gyrus or precuneus. TQ591's damage includes portions of the angular/intraparietal sulcus and precuneus, but do not impinge on the supramarginal gyrus. Because EE555 showed greater impairment, these data suggest that the angular gyrus may be particularly important for accessing autobiographical memories.

It is tempting to speculate that the memory retrieval deficit reflects a problem with internal attention. It has been known for over 50 years that parietal damage impairs various aspects of attention, most notably, the allocation of attention to spatial locations (Critchley, 1953). One conceptualization of simultanagnosia, a neurological disorder present in both of our patients for whom only a single item can be perceived at any time, is that it reflects a dramatic restriction of attention to a single object in space (for review, see Marshall and Fink, 2001). For instance, when shown a complex scene, they report individual components of the scene without gaining a comprehensive understanding of the picture. It is possible that this perceptual deficit is mirrored in an equally dramatic restriction of attention to internal representations, so that only a single piece or feature of any given memory is perceived. In other words, bilateral parietal damage may make it impossible to attend to and report on an entire memory, unless attention is readjusted or refocused by top-down cues such as the interviewer's pointed questions. In these patients, this type of attentional redirection is possible with visual stimuli. It is clear that the details of memories remain intact, because the patients are able to describe the information when probed. Thus, we suggest that the "pointers" to relevant information are inaccessible when the parietal lobe is damaged. The probe questions may serve as external pointers and thereby support the retrieval process.

This hypothesis also helps to explain why patients' recall of external details was somewhat impoverished under both free recall and probed recall conditions (Figs. 2, 3, 4). Because internal attention is too narrowly focused, patients may not suffer from the intrusions of other thoughts and memories that occur in neurologically normal individuals.

The present findings are not only consistent with functional neuroimaging evidence that PPC regions contribute to episodic memory retrieval but they also help clarify the nature of their contributions. The review by Wagner et al. (2005) considered three hypotheses regarding the role of parietal regions in episodic retrieval. The output buffer hypothesis states that the PPC holds retrieved information in a form accessible to decision-making processes, similarly to one of Baddeley's working memory buffers. The mnemonic accumulator hypothesis posits that PPC temporally integrates a memory-strength signal. The attention to internal representation hypothesis states that PPC shifts attention to, or maintains attention on, internally generated mnemonic representations. As noted by Wagner et al. (2005), each hypothesis can account for some, but not all, available evidence. To provide a more complete account of available evidence, we suggest a fourth hypothesis, which combines the third hypothesis with a distinction between top-down attention (attention guided by goals and intentions) and bottom-up attention (attention guided by incoming information). According to Corbetta and Shulman's (2002) attention model, top-down attention is medi- 
ated by dorsal PPC and bottom-up attention, by ventral PPC. Thus, the dual attentional processes hypothesis posits that dorsal PPC mediates top-down attentional processes controlling episodic retrieval according to task goals, whereas ventral PPC mediates bottom-up attentional processes that are captured by salient information recovered from episodic memory. Unlike other hypotheses, the dual attentional processes hypothesis can accommodate both evidence that dorsal PPC activity increases as a function of task demands (Dobbins et al., 2003; Dobbins and Wagner, 2005) and evidence that ventral PPC activity increases as a function of retrieval success (Yonelinas et al., 2005; Daselaar et al., 2006). This hypothesis is also consistent with the results of the current study. Given that both patients have ventral PPC lesions, the dual attentional processes hypothesis predicts they should have a deficit in spontaneously detecting information recovered from memory (bottom-up attention), but not in controlling retrieval processes guided by external prompts (top-down attention), which is what we found. Conversely, this hypothesis predicts that dorsal PPC lesions should impair the ability to modulate retrieval as guided by task demands, but not the capacity to spontaneously process memory contents. Although the attention to internal representation hypothesis could account for the present results as a general deficit in attention, it does not easily accommodate the difference between spontaneous versus prompted recall and does not predict a dissociation between episodic memory deficits in patients with lesions in dorsal versus ventral parietal lesions.

\section{Conclusion}

Bilateral parietal lobe damage impairs the ability to fully reexperience past autobiographical events. The memories described by such patients contain fewer details than those of normal control populations, although bilateral patients are not amnesic and had not previously been attributed with memory problems. General deficits in visual imagery do not explain the memory deficit, as the patients were able to accurately perform several visual imagery tasks. These findings indicate that normal parietal function is imperative for the effortless recollection of detailed episodic memories.

\section{References}

Addis DR, Moscovitch M, Crawley AP, McAndrews MP (2004) Recollective qualities modulate hippocampal activation during autobiographical memory retrieval. Hippocampus 14:752-762.

Baddeley A, Lewis V, Eldridge M, Thomson N (1984) Attention and retrieval from long-term memory. J Exp Psychol Gen 113:518-540.

Berryhill ME, Olson I (2007) Determining parietal involvement in visual working memory: causal or incidental? J Vis Abstr 7:300.

Bisiach E, Luzzatti C (1978) Unilateral neglect of representational space. Cortex 14:129-133.

Brooks LR (1968) Spatial and verbal components in the act of recall. Canadian J Psychol 22:349-368.

Byrne P, Becker S, Burgess N (2007) Remembering the past and imagining the future: a neural model of spatial memory and imagery. Psychol Rev 114:340-375.

Cabeza R, Nyberg L (2000) Neural bases of learning and memory: functional neuroimaging evidence. Curr Opin Neurol 13:415-421.

Cabeza R, St Jacques P (2007) Functional neuroimaging of autobiographical memory. Trends Cogn Sci 11:219-227.

Cabeza R, Rao SM, Wagner AD, Mayer AR, Schacter DL (2001) Can medial temporal lobe regions distinguish true from false? An event-related fMRI study of veridical and illusory recognition memory. Proc Natl Acad Sci USA 98:4805-4810.

Cavanna AE, Trimble MR (2006) The precuneus: a review of its functional anatomy and behavioural correlates. Brain 129:564-583.

Conway MA, Turk DJ, Miller SL, Logan J, Nebes RD, Meltzer CC, Becker JT
(1999) A positron emission tomography (PET) study of autobiographical memory retrieval. Memory 7:679-702.

Corbetta M, Shulman GL (2002) Control of goal-directed and stimulusdriven attention in the brain. Nat Rev Neurosci 3:201-215.

Craik FI, Dirkx E (1992) Age-related differences in three tests of visual imagery. Psychol Aging 7:661-665.

Craik FI, Govoni R, Naveh-Benjamin M, Anderson ND (1996) The effects of divided attention on encoding and retrieval processes in human memory. J Exp Psychol Gen 125:159-180.

Critchley M (1953) The parietal lobes. London: Edward Arnold.

Daselaar SM, Fleck MS, Cabeza RE (2006) Triple dissociation in the medial temporal lobes: recollection, familiarity, and novelty. J Neurophysiol 96:1902-1911.

De Renzi E, Nichelli P (1975) Verbal and nonverbal short-term memory impairment following hemispheric damage. Cortex 11:341-354.

D'Esposito M, Postle BR (1999) The dependence of span and delayedresponse performance on prefrontal cortex. Neuropsychologia 37:1303-1315.

Dobbins IG, Wagner AD (2005) Domain-general and domain-sensitive prefrontal mechanisms for recollecting events and detecting novelty. Cereb Cortex 15:1768-1778.

Dobbins IG, Rice HJ, Wagner AD, Schacter DL (2003) Memory orientation and success: separable neurocognitive components underlying episodic recognition. Neuropsychologia 41:318-333.

Eddy JK, Glass AL (1981) Reading and listening to high and low imagery sentences. J Verbal Learn Verbal Behav 20:333-345.

Eldridge LL, Knowlton BJ, Furmanski CS, Bookheimer SY, Engle SA (2000) Remembering episodes: a selective role for the hippocampus during retrieval. Nat Neurosci 3:1149-1152.

Farah MJ (1989) The neural basis of mental imagery. Trends Neurosci 12:395-399.

Farah MJ, Gazzaniga MS, Holtzman JD, Kosslyn SM (1985) A left hemisphere basis for visual mental imagery? Neuropsychologia 23:115-118.

Farah MJ, Hammond KM, Levine DN, Calvanio R (1988) Visual and spatial mental imagery: dissociable systems of representation. Cognit Psychol 20:439-462.

Ghent L, Mishkin M, Teuber HL (1962) Short-term memory after frontallobe injury in man. J Comp Physiol Psychol 55:705-709.

Gilboa A, Winocur G, Grady CL, Hevenor SJ, Moscovitch M (2004) Remembering our past: functional neuroanatomy of recollection of recent and very remote personal events. Cereb Cortex 14:1214-1225.

Hassabis D, Kumaran D, Vann SD, Maguire EA (2007) Patients with hippocampal amnesia cannot imagine new experiences. Proc Natl Acad Sci USA 104:1726-1731.

Henson RN, Rugg MD, Shallice T, Josephs O, Dolan RJ (1999) Recollection and familiarity in recognition memory: an event-related functional magnetic resonance imaging study. J Neurosci 19:3962-3972.

Husain M, Mannan S, Hodgson T, Wojciulik E, Driver J, Kennard C (2001) Impaired spatial working memory across saccades contributes to abnormal search in parietal neglect. Brain 124:941-952.

Johnson MK, Foley MA, Suengas AG, Raye CL (1988) Phenomenal characteristics of memories for perceived and imagined autobiographical events. J Exp Psychol Gen 117:371-376.

Konishi S, Wheeler ME, Donaldson DI, Buckner RL (2000) Neural correlates of episodic retrieval success. NeuroImage 12:276-286.

Kopelman MD, Wilson BA, Baddeley AD (1989) The autobiographical memory interview: a new assessment of autobiographical and personal semantic memory in amnesic patients. J Clin Exp Neuropsychol 11:724-744.

Kosslyn SM, Holtzman JD, Farah MJ, Gazzaniga MS (1985) A computational analysis of mental image generation: evidence from functional dissociations in split-brain patients. J Exp Psychol Gen 114:311-341.

Legendre P, Oden NL, Sokal RR, Vaudor A, Kim J (1990) Approximate analysis of variance of spatially autocorrelated regional data. J Classification 7:53-75.

Levine B (2004) Autobiographical memory and the self in time: brain lesion effects, functional neuroanatomy, and lifespan development. Brain Cogn 55:54-68.

Levine B, Svoboda E, Hay JF, Winocur G, Moscovitch M (2002) Aging and autobiographical memory: dissociating episodic from semantic retrieval. Psychol Aging 17:677-689. 
Levine B, Turner GR, Tisserand D, Hevenor SJ, Graham SJ, McIntosh AR (2004) The functional neuroanatomy of episodic and semantic autobiographical remembering: a prospective functional MRI study. J Cogn Neurosci 16:1633-1646.

Levine DN, Warach J, Farah M (1985) Two visual systems in mental imagery: dissociation of "what" and "where" in imagery disorders due to bilateral posterior cerebral lesions. Neurology 35:1010-1018.

Luria AR (1966) The higher cortical functions in man. New York: Basic Books.

Maguire EA, Mummery CJ (1999) Differential modulation of a common memory retrieval network revealed by positron emission tomography. Hippocampus 9:54-61.

Malhotra P, Jager HR, Parton A, Greenwood R, Playford ED, Brown MM, Driver J, Husain M (2005) Spatial working memory capacity in unilateral neglect. Brain 128:424-435.

Manly BF (1997) Randomization, bootstrap, and Monte Carlo methods in biology, second edition. London: Chapman and Hall.

Marshall JC, Fink GR (2001) Spatial cognition: where we were and where we are. NeuroImage 14:S2-S7.

Nyberg L, Forkstam C, Petersson KM, Cabeza R, Ingvar M (2002) Brain imaging of human memory systems: between-systems similarities and within-system differences. Brain Res Cogn Brain Res 13:281-292.

Paivio A (1978) Comparisons of mental clocks. J Exp Psychol Hum Percept Perform 4:61-71.

Pisella L, Berberovic N, Mattingley JB (2004) Impaired working memory for location but not for colour or shape in visual neglect: a comparison of parietal and non-parietal lesions. Cortex 40:379-390.

Risse GL, Rubens AB, Jordan LS (1984) Disturbances of long-term memory in aphasic patients. A comparison of anterior and posterior lesions. Brain 107:605-617.

Rosenbaum RS, McKinnon MC, Levine B, Moscovitch M (2004) Visual imagery deficits, impaired strategic retrieval, or memory loss: disentangling the nature of an amnesic person's autobiographical memory deficit. Neuropsychologia 42:1619-1635.

Svoboda E, McKinnon MC, Levine B (2006) The functional neuroanatomy of autobiographical memory: a meta-analysis. Neuropsychologia 44:2189-2208.

Tippett LJ (1992) The generation of visual images: a review of neuropsychological research and theory. Psychol Bull 112:415-432.

Wagner AD, Shannon BJ, Kahn I, Buckner RL (2005) Parietal lobe contributions to episodic memory retrieval. Trends Cogn Sci 9:445-453.

Warrington EK, Logue V, Pratt RT (1971) The anatomical localisation of selective impairment of auditory verbal short-term memory. Neuropsychologia 9:377-387.

Wheeler ME, Buckner RL (2004) Functional-anatomic correlates of remembering and knowing. NeuroImage 21:1337-1349.

Wojciulik E, Husain M, Clarke K, Driver J (2001) Spatial working memory deficit in unilateral neglect. Neuropsychologia 39:390-396.

Yonelinas AP, Otten LJ, Shaw KN, Rugg MD (2005) Separating the brain regions involved in recollection and familiarity in recognition memory. J Neurosci 25:3002-3008. 